\title{
Pautas de crianza y desarrollo socioafectivo
}

\author{
Gemma CARDERA GIL \\ al192553@uji.es \\ FRANCISCO JUAN GARCÍA BACETE \\ fgarcia@uji.es
}

\section{Resumen}

Introducción: La presente investigación examina la relación entre los estilos parentales de los padres (autoritario, permisivo y democrático) y la competencia socioemocional del niño. Método: La muestra está compuesta por 160 alumnos de $6 .^{\circ}$ de primaria (76 niños y 84 niñas). La información ha sido proporcionada por diversos informantes: las madres cumplimentaron el cuestionario de estilos y dimensiones parentales; los profesores, el cuestionario de cualidades y dificultades y los alumnos, el cuestionario sociométrico de preferencias. Se han realizado correlaciones, tablas de contingencias y regresiones. Resultados: Los resultados indican que el estilo educativo democrático se asocia significativamente con la prosocialidad del niño, al contrario que los estilos permisivo y autoritario, y que este último, a su vez, se relaciona negativamente y de manera significativa con problemas de conducta e hiperactividad. En cuanto al género, no se encuentra relación entre el sexo de los niños y niñas y el estilo educativo empleado para cada uno de ellos. Por otro lado, relacionando los estilos educativos parentales con la aceptación por parte de los compañeros de clase, se encuentra que los niños cuyos padres utilizan un estilo autoritario reciben más nominaciones negativas por parte de sus compañeros, mientras que aquellos niños educados en un estilo democrático o permisivo reciben mayor número de nominaciones positivas. Conclusión: Los resultados sugieren que, para fomentar la buena sociabilidad de los niños y niñas y la aceptación entre iguales, es importante un adecuado estilo educativo dentro de las familias, sugiriendo para ello programas de formación de madres y padres.

Palabras clave: estilo educativo, desarrollo, conducta, sociabilidad.

\section{Abstract}

Introduction: This research examines the relationship between parenting styles (authoritarian, permissive and democratic) and the child's socio-emotional competence. Method: The sample was composed of 160 year- 6 primary school students (76 boys and 84 girls). Information was obtained with the following measuring instruments: (Parenting Styles and Dimensions Questionnaire (PSDQ); Strengths and Difficulties Questionnaires (SDQ) and the Sociometry Questionnaire). Correlations, contingency tables and regressions were made. Results: The results indicate that a democratic style of education is significantly associated with child prosociality, unlike the authoritarian and permissive styles, the latter of which is negatively and significantly 
associated with behavioural problems and hyperactivity. No relationship between children's gender and education style was found. After analysing parenting styles with acceptance by classmates, the results showed that the children whose parents used an authoritarian style received more negative nominations by their classmates, while those raised according to democratic or permissive styles received many more positive nominations. Conclusion: The results suggest that in order to promote good children socialisation and acceptance by peers, an adequate education style in families is important. For this reason, training programmes are suggested for mothers and fathers.

Keywords: Parenting styles, development, behaviour, sociability

\section{Introducción}

El presente trabajo pretende contribuir a la comprensión de la relación entre los estilos educativos parentales y el desarrollo socioafectivo del niño a través de un estudio realizado desde el Departamento de Psicología Evolutiva, Educativa, Social y Metodología de la Universitat Jaume I de Castellón.

Tras una revisión teórica acerca del desarrollo socioafectivo en la infancia, se expone la investigación en la cual han participado 200 alumnos de $6 .^{\circ}$ de primaria (edades comprendidas entre 11 y 12 años) de la provincia de Castellón, con tal de explicar, de manera empírica, la influencia que tienen las pautas de crianza y la educación parental en el desarrollo cognitivo y social del niño, otorgando así la importancia que merece el núcleo familiar desde la primera infancia.

El desarrollo infantil, entendido como un conjunto de sucesos y cambios físicos y psicológicos a lo largo de toda la infancia, viene influenciado por diversos factores, tanto genéticos como ambientales, entre ellos, los estilos educativos, los cuales regulan el desarrollo cognitivo y social del niño estableciendo pautas de comportamiento, los estilos de aprendizaje y las diferencias individuales, evidenciando que las relaciones sociales son un pilar básico para el crecimiento del infante y, tal y como informan Denham, von Salisch, Olthof, Kochanoff y Caverly (2002), las expresiones emocionales y afectivas que se ofrecen a los bebés tienen funciones reguladoras en sus interacciones sociales.

Las primeras interacciones sociales que establece el niño son con sus cuidadores, asentando así las bases de vinculaciones posteriores, influyendo en la manera de interaccionar con los demás, la percepción de uno mismo y la comprensión del mundo social, adoptando pautas de crianza distintas a la hora de educar a un hijo (Palacios y Moreno, 1994). Entre las definiciones de estilos educativos, la que más apoyo empírico ha obtenido es la de Darling y Steinberg (1993), los cuales los definieron como un conjunto de actitudes paternales hacia los hijos, presentados mediante conductas que originan un clima emocional e incluyen hábitos educativos o de cuidado, gestos, tono de voz, lenguaje corporal o expresiones emocionales espontáneas.

Diana Baumrind (1978), autora pionera de los estudios sobre pautas de crianza y desarrollo social, influenciada por Kurt Lewin, diferencia tres variables (comunicación, control e implicación afectiva) y establece tres tipos de estilos educativos parentales:

Autoritario. Estos padres basan su educación en las normas y el orden. La obediencia es fundamental para ellos, rechazan el diálogo y adoptan el castigo y la subordinación de los 
hijos como medida disciplinaria. Mantienen un estilo educativo jerárquico en el cual la comunicación es de arriba-abajo, dejando a los niños en un nivel inferior e incapacitándolos de autonomía personal y creativa.

Permisivo. Estilo educativo basado en la tolerancia y la permisividad de los impulsos de los niños, no aceptan ningún tipo de castigo como disciplina ni otorgan tareas ni obligaciones a los hijos, alejándolos de las responsabilidades, los límites y las normas.

Democrático/Autoritativo. Educación basada en la imposición de roles y tareas mediante el diálogo y la negociación niño-adulto, se trata de una jerarquía bidireccional de mutuo respeto en la cual ambas partes son conscientes de los deberes y derechos propios y del otro.

A partir de estos estilos, diversos estudios analizan la relación entre los estilos educativos de los padres con el posterior desarrollo del infante, creando así correlaciones que puedan justificar el mejor patrón de crianza dentro de una familia.

Palacios y Moreno (1994) afirman en su estudio que aquellos hijos e hijas de padres y madres con un estilo democrático presentan características más deseables como alta autoestima, constancia a la hora de realizar tareas, confianza para afrontar nuevas situaciones, adecuada competencia social, autocontrol y buena capacidad para interiorizar los valores sociales y morales de la sociedad.

La revisión realizada por Eisenberg, Spinrad y Knafo-Noam (2015) concluyó que la calidez de los padres, la capacidad de respuesta, el apoyo y la participación con sus hijos se relacionan positivamente con el comportamiento prosocial de los niños.

Finalmente, el estudio llevado a cabo por Pastorelli y cols. (2015) se centró en ocho países distintos para dar a conocer la influencia de un estilo parental positivo respecto el comportamiento prosocial de los hijos, utilizando los instrumentos Quality of mother-child relationship (child-reports), Balanced positive parental discipline (child-reports) y Children's prosocial behavior (mother and child-report). Mediante dicha investigación, confirmó que la parentalidad positiva daba como resultado una conducta prosocial en los hijos y que esto ocurre mayormente en la infancia.

El objetivo principal del presente trabajo empírico es analizar la relación entre los diferentes estilos educativos definidos por Diana Baumrind y el ajuste social y emocional del niño y sus relaciones de aceptación y rechazo con iguales, ofreciendo así resultados más recientes a la investigación.

\section{Método}

La muestra ha sido elegida de manera aleatoria y está compuesta por 203 alumnos de $6 .^{\circ}$ de primaria de varios colegios de la provincia de Castellón.

Para acceder a ella, se pidió permiso a la Generalitat Valenciana para que permitiera realizar un estudio longitudinal y administrar diversos cuestionarios en los centros educativos. Una vez conseguida la autorización de Conselleria de Educación, se informó a los centros de la investigación y sus objetivos y se facilitó una autorización a los padres y madres para pasar los cuestionarios, ya que se debía obtener un $75 \%$ o más de firmas para poder llevarlo a cabo. De igual modo, se garantizó total confidencialidad, tanto en los datos de los centros como de los propios alumnos.

Los instrumentos de evaluación administrados han sido los siguientes: 
1) Cuestionario sociométrico de preferencias (García Bacete y cols., 2014).

- Instrumento utilizado para conocer la relación entre iguales dentro del aula a partir de un sistema de nominaciones que consiste en que los niños elijan a los compañeros a partir de dos criterios diferentes: el positivo y el negativo. De igual modo, aparte de evaluar los criterios de los niños sobre el resto, se evalúa también la percepción subjetiva que estos tienen de lo que piensan el resto respecto de ellos mismos.

- Los resultados se miden a partir del software Sociomet, el cual proporciona los tipos sociométricos de las aulas (siendo estos los grupos de preferidos, rechazados, ignorados, controvertidos y promedio) y otras variables importantes como nominaciones positivas emitidas (NPE), nominaciones negativas emitidas (NNE), impresión positiva (IP), impresión negativa (IN), reciprocidad positiva (RP), reciprocidad negativa (RN), oposición de sentimientos (OS), nominaciones positivas recibidas (NPR), nominaciones negativas recibidas (NNR), percepciones positivas (PP) y percepciones negativas (PN).

2) Cuestionario de cualidades y dificultades (SDQ) (Goodman, 1997)

- Cuestionario utilizado para estudiar problemas de comportamiento en la versión para el profesorado. El instrumento consta de 25 ítems divididos en 5 escalas de 5 ítems cada una.

- Cuatro de las cinco escalas miden conductas problemáticas (síntomas emocionales, problemas de conducta, hiperactividad y problemas con los compañeros) y la quinta escala del cuestionario entero, denominada escala total del dificultades, mide la conducta prosocial.

- Las respuestas a cada ítem se realizan mediante una escala del 0 a $2(0=$ no es verdad; 1 = verdad a medias y 2 = absolutamente cierto), obteniendo así, en cada escala, una puntuación comprendida entre el 0 y el 10, exceptuando la escala total de dificultades que comprende una puntuación del 0 al 40, sumándose los resultados de cuatro de las cinco escalas, ya que la última (conducta prosocial) no interviene.

3) Cuestionario de estilos y dimensiones parentales (PSDQ, Parenting Styles and Dimensions Questionnaire, Robinson, Mandleco, Olsen y Hart, 2001)

- La escala está compuesta por 52 ítems divididos en tres subescalas, correspondientes a los estilos parentales descritos anteriormente (democrático, autoritario y permisivo). A su vez, cada una de estas se compone de otras subescalas más específicas, de tal modo que la escala democrática se divide en afecto/implicación (por ejemplo, expreso afecto a mi hijo/a con abrazos y besos); razonamiento/inducción (por ejemplo, le explico las consecuencias de su conducta); participación democrática (por ejemplo, le animo a expresarse libremente incluso cuando está en desacuerdo conmigo) y carácter/trato relajado (por ejemplo, le explico cómo me siento cuando se porta bien y cuando se porta mal). La escala autoritaria está compuesta por hostilidad verbal (por ejemplo, regaño y critico a mi hijo para que mejore); castigo físico (por ejemplo, uso el castigo físico para educarlo/a); estrategias punitivas / no razonamiento (le castigo dejándolo/a solo/a en algún lugar sin darle explicaciones) y directividad autoritaria (por ejemplo, le digo lo que ha de hacer). Y, finalmente, la escala permisiva se divide en falta de supervisión (por ejemplo, le permito que interrumpa a otros); ig- 
norar el mal comportamiento (por ejemplo, ignoro su mala conducta) y falta de autoconfianza como padre/madre (por ejemplo, doy la impresión de estar inseguro/a sobre cómo resolver su mala conducta).

\section{Resultados}

\section{Correlaciones}

Se midió si los estilos educativos de los padres tenían relación con las conductas de los niños (tabla 1) y con las nominaciones que estos recibían en clase (tabla 2). Para dicho análisis se realizaron correlaciones.

Tabla 1

Estilos educativos parentales y conductas de los niños

\begin{tabular}{|c|c|c|c|c|c|}
\hline \multirow[t]{3}{*}{ SDQ } & & SÍNTOMAS & PROBLEMAS & & PROSOCIALIDAD \\
\hline & & & & & \\
\hline & Correlación de Pearson &,- 003 & -164 &,- 111 & $295^{* *}$ \\
\hline \multirow[t]{2}{*}{ DEMOCRÁTICO } & Sig- (bilateral) & 971 & 051 & .186 & 000 \\
\hline & $\mathrm{N}$ & 143 & 143 & 143 & 143 \\
\hline \multirow[t]{3}{*}{ AUTORITARIO } & Correlación de Pearson & 063 & $260^{*}$ & 059 & $-245^{* *}$ \\
\hline & Sig. (bilateral) & 454 &, 002 & 483 &, 003 \\
\hline & $\mathrm{N}$ & 143 & 143 & 143 & 143 \\
\hline \multirow[t]{2}{*}{ PERMISIVO } & Correlación de Pearson & 001 & 081 & 075 & $-217^{* *}$ \\
\hline & Sig. (bilateral) & 990 & 335 & 374 &, 009 \\
\hline
\end{tabular}

El estilo educativo democrático se relaciona negativamente con síntomas emocionales, problemas de conducta y problemas con los compañeros, mientras que correlaciona positiva y significativamente al $99 \%$ con la prosocialidad del niño.

El estilo autoritario correlaciona con una significación del $99 \%$ y de manera positiva con los problemas de conducta, mientras que se relaciona de manera negativa con la prosocialidad.

El estilo permisivo correlaciona de manera negativa y con una significación del $99 \%$ con la prosocialidad, mientras que está relacionado positivamente con los síntomas emocionales, problemas de conducta y problemas con los compañeros. 
Tabla 2

Estilos educativos y nominaciones recibidas en clase

\begin{tabular}{llrr}
\hline \multirow{2}{*}{ DEMOCRÁTICO } & Correlación de Pearson & $\begin{array}{c}\text { NPR/N-1 } \\
\text { Nominaciones } \\
\text { Positivas Recibidas }\end{array}$ & $\begin{array}{c}\text { NNR/N-1 } \\
\text { Nominaciones } \\
\text { Negativas Recibidas }\end{array}$ \\
& Sig. (bilateral) &, 044 &,- 015 \\
& N &, 600 &, 856 \\
AUTORIT ARIO & Correlación de Pearson & 143 & 143 \\
& Sig. (bilateral) &,- 109 &, 056 \\
& N &, 195 &, 509 \\
PERMISIVO & Correlación de Pearson & 143 & 143 \\
& Sig. (bilateral) &, 006 &, 012 \\
& N &, 941 &, 883 \\
& & 143 & 143 \\
\hline
\end{tabular}

El estilo democrático se relaciona positivamente con las nominaciones positivas que recibe el niño o la niña en clase por sus compañeros y de manera negativa con las nominaciones negativas, mientras que el estilo autoritario correlaciona negativamente con las nominaciones positivas y viceversa.

\section{Tablas de contingencia}

Se midió si los estilos educativos de los padres tenían relación con el sexo de los niños y niñas de la muestra, utilizando el procedimiento de tablas cruzadas. Los resultados indican que no hay diferencias significativas, es decir, que los padres informan de las mismas proporciones de estilos educativos en hijos varones y hembras.

\section{Discusión y conclusiones}

Los estilos educativos están relacionados con las conductas socioafectivas de los niños, confirmando así los resultados encontrados por Eisenberg y cols. (2015).

El estilo educativo democrático se asocia significativamente con la prosocialidad del niño, al contrario que los estilos permisivo y autoritario, y este último, a su vez, se relaciona negativamente y de manera significativa con problemas de conducta, de modo que se confirman los resultados obtenidos por Pastorelli y cols. (2015). Los niños cuyos padres utilizan un estilo autoritario reciben más nominaciones negativas por parte de sus compañeros, mientras que aquellos niños educados en un estilo democrático reciben mayor número de nominaciones positivas.

Según informan los padres, el estilo empleado por estos no se ve relacionado con el género de los niños, de tal modo que se encuentran los mismos porcentajes de estilos independientemente de que el hijo sea chico o chica, es decir, los padres no son más autoritarios con las chicas o más permisivos con los chicos, etc. 
A partir de estos resultados, creemos que, para fomentar la buena sociabilidad de niños y niñas y la aceptación entre iguales, es importante un adecuado estilo educativo dentro de las familias, siendo el estilo democrático, que tiene como base la promoción de la comunicación bidireccional, el que mejores resultados obtiene. Para promover este estilo democrático se sugiere la formación de buenos profesionales y el desarrollo de programas de formación de madres y padres y de cooperación entre familias y escuelas.

\section{Referencias bibliográficas}

Baumrind, D. (1978). Parental disciplinary patterns and social competence in children. Youth and Society, 9, 239-276.

Darling, N. y Steinberg, L. (1993). Parenting style as context: An integrative model. Psychological Bulletin, 113, 487-496.

Denham, S., von Salisch, M., Olthof, T., Kochanoff, A. y Caverly, S. (2002). Emotional and social development in childhood. En P. K. Smith y C. H. Hart (eds.), Blackwell, Handbook of childhood social development (pp. 307-328). UK: Blackwell.

Eisenberg, N., Spinrad, T. L., y Knafo-Noam, A. (2015). Prosocial development. En M. E. Lamb y C. Garcia Coll (vol. eds.), R. M. Lerner (series ed.), Handbook of child psychology, vol. 3, Social, emotional, and personality development (7th edn, pp. 610-656). New York: Wiley.

García Bacete, F. J., Jiménez, I., Muñoz Tinoco, V., Marande, G., Monjas, M. ${ }^{a}$ L., Sureda, I. y cols. (2014). El rechazo entre iguales en su contexto interpersonal: Una investigación con niños y niñas de primer ciclo de primaria. Castellón: Davalos-Fletcher.

Goodman, R. (1997). Cuestionario de Cualidades y Dificultades: A Research Note. Journal of Child Psychology and Psychiatry, 38, 581-586.

Palacios, J. y Moreno, M. C. (1994). Contexto familiar y desarrollo social. En M. J. Rodrigo (ed.), Contexto y Desarrollo social (pp. 157-188). Madrid: Síntesis.

Pastorelli, C., Lansford, J. E., Luengo Kanacri, B. P., Malone, P. S., Di Giunta, L., Bacchini, D. y cols. (2015). Positive parenting and children's prosocial behavior in eight countries. Journal of Child Psychology and Psychiatry and Allied Disciplines. doi:10.1111/ jcpp. 12477

Robinson, C. C.; Mandleco, B., Olsen, S. F. y Hart, C. H. (2001). Cuestionario de estilos y dimensiones parentales (PSDQ). En B. F. Perlmutter, J. Touliatos y G. W. Holden (eds.), Handbook of family measurement techniques. Vol. 2: Instruments and Index (pp. 97123). Thousand Oaks, CA: Sage. 